\title{
Hot topic: alignment in total knee arthroplasty—systematic versus more individualised alignment strategies
}

\author{
Michael T. Hirschmann ${ }^{1,2} \cdot$ Jon Karlsson ${ }^{3} \cdot$ Roland Becker $^{4}$
}

Received: 26 March 2018 / Accepted: 18 April 2018 / Published online: 21 April 2018

(c) European Society of Sports Traumatology, Knee Surgery, Arthroscopy (ESSKA) 2018

Keywords Total knee arthroplasty · TKA · Total knee replacement · TKR · Knee · Alignment · Kinematic alignment · Mechanical alignment $\cdot$ Anatomic alignment $\cdot$ Personalised medicine

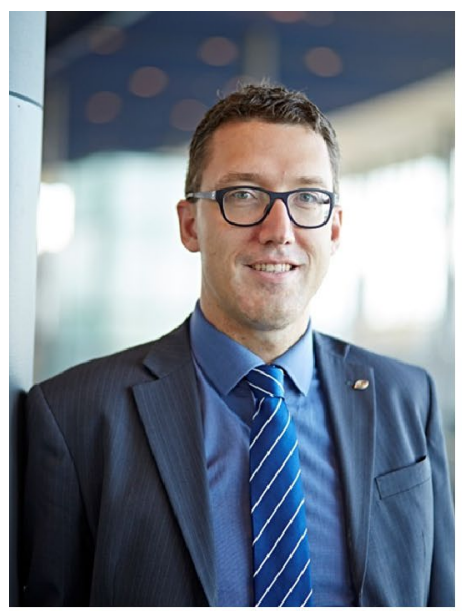

Michael T. Hirschmann

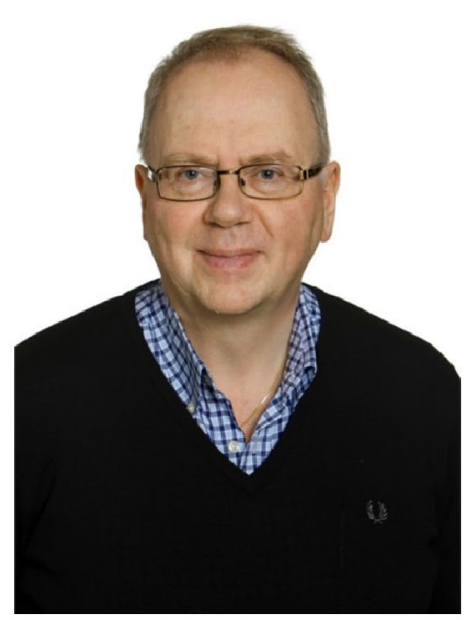

Jon Karlsson

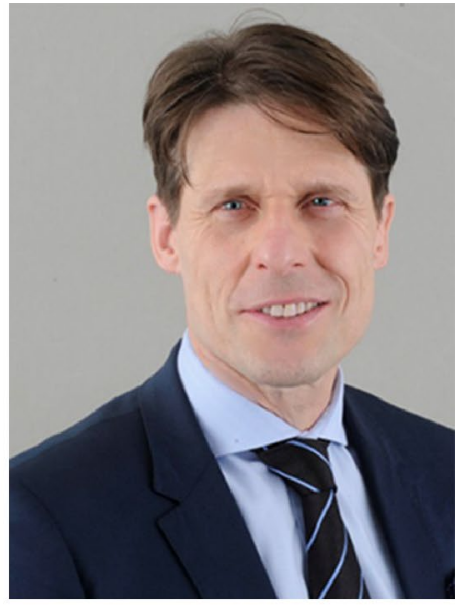

Roland Becker
Alignment is regarded as a hot topic in total knee arthroplasty (TKA) [3]. For a long time, a neutral mechanical alignment was proposed as the only reliable, durable option in TKA [2]. With the increasing quality and durability of polyethylene inlays, many knee surgeons are increasingly

Michael T. Hirschmann

Michael.Hirschmann@unibas.ch

1 Department of Orthopaedic Surgery and Traumatology, Kantonsspital Baselland (Bruderholz, Liestal, Laufen), 4101 Bruderholz, Switzerland

2 University of Basel, Basel, Switzerland

3 Department of Orthopaedics, Sahlgrenska University Hospital, Sahlgrenska Academy, Gothenburg University, Göteborg, Sweden

4 Department of Orthopaedic and Traumatology, Brandenburg Medical School Theodor Fontane, Hochstrasse 29, 14770 Brandenburg/Havel, Germany gaining or regaining interest in alternative alignment methods such as anatomic or kinematic alignment [1]. Some authors are even openly questioning the concept of mechanical alignment [1]. In fact, this is accompanied by a more profound understanding of the distribution of knee alignment among patient populations. In particular, the morphology and asymmetry of the distal femur has been investigated in several studies.

When it comes to alignment, systematic alignment can be differentiated from more individualised alignment strategies [3]. A systematic approach means that this alignment strategy is used in every patient homogeneously [3]. Currently, a systematic mechanical alignment strategy is most widely used by those of us who are knee surgeons.

In contrast, a more individualised alignment strategy would adapt the alignment strategy with regard to the individual constitutional alignment of each patient [3]. It aims to restore the individual patient alignment. However, there are 
a considerable number of open questions. To date, knowledge of the distribution of knee alignment in large-scale populations is still not sufficient. Furthermore, it is unclear whether the individual morphology and alignment geometry changes either over the years or due to osteoarthritis.

This issue of KSSTA focuses on alignment in TKA and in particular highlights three papers which aim to shed some light on this topic.

In a cadaver study, Roth et al. investigated tibial forces, differences in tibial forces between medial and lateral compartments, and anterior translation of the contact locations of the femoral component on the tibial component during passive flexion in cruciate-retaining, kinematically aligned TKA [4]. The authors found that average total tibial forces, average differences in tibial forces between compartments, and posterior translation of the tibial contact locations were similar to those in native knees [4]. The authors concluded that kinematically aligned TKA limits high tibial forces, differences in tibial forces between compartments, and anterior translation of the tibial contact locations during passive flexion [4].

However, one major concern that still remains is how much varus in the tibial component is acceptable without compromising survival rates in TKA [7]. This concern is fuelled by a recent landmark long-term RSA study by Teeter et al., which examined implant migration using RSA [6]. The aim was neutral mechanical alignment. At the 10-year follow-up after posterior stabilised TKA, it was seen that implant migration increased with an increasing varus tibial alignment [6]. Condylar liftoff was far more common in the varus group [6]. The authors concluded that the increased migration and liftoff raise concerns about the longevity of malaligned TKA. The tibial component should, therefore, be neutrally aligned [6].

Finally, Slevin et al. assessed the relationship between coronal TKA alignment using 3D reconstructed CTs and clinical outcome in patients with preoperative varus in comparison to patients with natural or valgus deformity [5]. The authors found that, in the preoperatively non-varus group, a highly significant correlation was found between neutral limb alignment and better KSS [5]. In the varus group, no correlation was found between the postoperative limb alignment and the position of components in the coronal plane and KSS score [5]. The authors concluded that the concept of constitutional varus alignment is still the subject of debate [5]. Moreover, it appears that the objective should be a more individualised alignment target based on the individual knee phenotype [5].

To summarise, this is an exciting time, as our knowledge of the variability of individual knee morphology is increasing every day and the current alignment strategies in TKA are under investigation. Time will tell if the more personalised medicine for TKA alignment leads to better outcomes and fewer dissatisfied patients after TKA.

Funding There was no funding

\section{Compliance with ethical standards}

Conflict of interest The authors delcare that there is no conflict of interest.

Ethical approval This article does not contain any studies with human participants performed by any of the authors.

Informed consent For this type of study formal consent is not required.

\section{References}

1. Lee YS, Howell SM, Won YY, Lee OS, Lee SH, Vahedi H, Teo SH (2017) Kinematic alignment is a possible alternative to mechanical alignment in total knee arthroplasty. Knee Surg Sports Traumatol Arthrosc 25(11):3467-3479

2. Longstaff LM, Sloan K, Stamp N, Scaddan M, Beaver R (2009) Good alignment after total knee arthroplasty leads to faster rehabilitation and better function. J Arthroplast 24(4):570-578

3. Riviere C, Iranpour F, Auvinet E, Howell S, Vendittoli PA, Cobb J, Parratte S (2017) Alignment options for total knee arthroplasty: a systematic review. Orthop Traumatol Surg Res 103(7):1047-1056

4. Roth JD, Howell SM, Hull ML (2017) Kinematically aligned total knee arthroplasty limits high tibial forces, differences in tibial forces between compartments, and abnormal tibial contact kinematics during passive flexion. Knee Surg Sports Traumatol Arthrosc. https://doi.org/10.1007/s00167-017-4670-Z

5. Slevin O, Hirschmann A, Schiapparelli FF, Amsler F, Huegli RW, Hirschmann MT (2017) Neutral alignment leads to higher knee society scores after total knee arthroplasty in preoperatively non-varus patients: a prospective clinical study using 3D-CT. Knee Surg Sports Traumatol Arthrosc. https://doi.org/10.1007/s00167-017-4744-y

6. Teeter MG, Naudie DD, McCalden RW, Yuan X, Holdsworth DW, MacDonald SJ, Lanting BA (2017) Varus tibial alignment is associated with greater tibial baseplate migration at 10 years following total knee arthroplasty. Knee Surg Sports Traumatol Arthrosc. https://doi.org/10.1007/s00167-017-4765-6

7. Vandekerckhove PTK, Teeter MG, Naudie DDR, Howard JL, MacDonald SJ, Lanting BA (2017) the impact of coronal plane alignment on polyethylene wear and damage in total knee arthroplasty: a retrieval study. J Arthroplast 32(6):2012-2016 